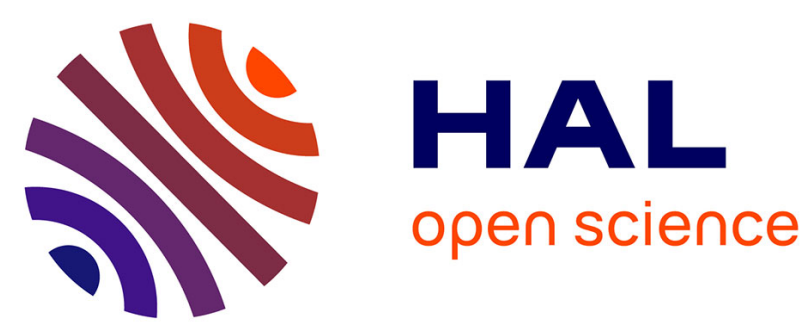

\title{
Rate-distortion optimization of a tone mapping with SDR quality constraint for backward-compatible high dynamic range compression
}

David Gommelet, Aline Roumy, Christine Guillemot, Michael Ropert, Julien Letanou

\section{To cite this version:}

David Gommelet, Aline Roumy, Christine Guillemot, Michael Ropert, Julien Letanou. Rate-distortion optimization of a tone mapping with SDR quality constraint for backward-compatible high dynamic range compression. International Conference on Image Processing, ICIP, Sep 2016, Phoenix, United States. pp.1384-1388, 10.1109/ICIP.2016.7532585 . hal-01377701

\section{HAL Id: hal-01377701 \\ https://hal.inria.fr/hal-01377701}

Submitted on 7 Oct 2016

HAL is a multi-disciplinary open access archive for the deposit and dissemination of scientific research documents, whether they are published or not. The documents may come from teaching and research institutions in France or abroad, or from public or private research centers.
L'archive ouverte pluridisciplinaire HAL, est destinée au dépôt et à la diffusion de documents scientifiques de niveau recherche, publiés ou non, émanant des établissements d'enseignement et de recherche français ou étrangers, des laboratoires publics ou privés. 


\title{
RATE-DISTORTION OPTIMIZATION OF A TONE MAPPING WITH SDR QUALITY CONSTRAINT FOR BACKWARD-COMPATIBLE HIGH DYNAMIC RANGE COMPRESSION
}

\author{
David Gommelet $^{\dagger \star}$, Aline Roumy ${ }^{\star}$, Christine Guillemot $^{\star}$, Michael Ropert $^{\dagger}$, Julien LeTanou ${ }^{\dagger}$ \\ ${ }^{\dagger}$ Envivio. Inc, Saint Jacques de La Lande, France (initial+lastname@envivio.com) \\ * Inria Rennes-Bretagne-Atlantique, Rennes, France (firstname.lastname@inria.fr)
}

\begin{abstract}
This paper addresses the problem of designing a global tone mapping operator for rate-distortion optimized backward compatible compression of HDR images. We consider a two layer coding scheme in which a base SDR layer is coded with HEVC, inverse tone mapped and subtracted from the input HDR signal to yield the enhancement HDR layer. The tone mapping curve design is formulated as the minimization of the distortion on the reconstructed HDR signal under the constraint of a total rate cost on both layers, while preserving a good quality for the SDR signal. We first demonstrate that the optimum tone mapping function only depends on the rate of the base SDR layer and that the minimization problem can be separated in two consecutive minimization steps. Experimental results show that the proposed tone mapping optimization yields the best trade-off between rate-distortion performance and quality preservation of the coded SDR.
\end{abstract}

Index Terms - High dynamic range (HDR), Compression, Tone-Mapping (TMO), Backward-Compatible, HEVC

\section{INTRODUCTION}

Standard Dynamic Range (SDR) color spaces encode a small fraction of the visible color gamut for luminance values ranging from 0.1 to $100 \mathrm{~cd} / \mathrm{m}^{2}$, while the human eye can see luminance values going from $10^{-6}$ to $10^{8}$ with adaptation of the pupil [1] and around 5 orders of magnitude without pupil adaptation [2]. HDR imaging, with raw data represented with floating point numbers, aims at capturing all the luminance values which the human eye can perceive. For efficient coding, storage and manipulation, this floating point data is first perceptually encoded into integer values using Perceptual Quantizer Transfer Functions (PQTFs). Several formats exist for the perceptually encoded HDR data such as the RGBE [3] format and the OpenEXR [4]. In order to use legacy video codecs to compress these HDR video formats, the data needs to be further transformed as in [5] where the logluv [6] transform is used to convert the OpenEXR files in 14 bits for the luma representation and 8 bits for color components. In [7], the SMPTE-2084 [8] is used to transform the HDR content into 10 bits before encoding it with HEVC.

To be visualized on legacy displays, the HDR content needs to be tone mapped. Many tone mapping operators
(TMO) have been developed to convert the HDR content into SDR content with the goal of preserving the perception of the content or the artistic intent. One example of TMO is the photographic tone reproducer (PTR) [9], shown to be one of the two TMO that consistently perform well and to be the best in terms of visual quality and image integrity $[10,11]$.

In this paper, we consider the problem of SDR backward compatible coding of HDR content with two layers. Note that this problem has already been addressed in the literature. In [12], the SDR image and a ratio image between the SDR and HDR images are encoded using JPEG. Only the SDR image can be decoded, or both to provide the HDR version. To improve the coding performance, the authors in [13] use an inverse TMO instead of a ratio image. While the above TMO aim at preserving the perception and artistic intent when converting the content, one can also try to design the TMO in order to optimize the compression efficiency $[14,15,16]$. The authors in [14] design a TMO parameterized by a piecewise linear tone-curve which minimizes the MSE between the logarithmic values $(\log 10)$ of the input and the inverse tone mapped HDR luminance values.

In [15], the authors notice that minimizing the MSE can increase the spatial complexity of the generated SDR. The approach of [14] is then extended in [15] and [16] in order to minimize a rate-distortion (R-D) cost, considering the rate cost of the SDR layer only.

Optimizing the TMO only for compression purposes may yield SDR content in which the "perception" and artistic intent of the HDR content is not preserved [17, 18]. This observation also applies to the proposals [19] and [20] to the MPEG CFE [7]. These proposals are backward compatible but the perceptual quality of the SDR signal is not constrained to a reference. However, they judge their SDR to be of sufficient perceptual quality.

The approach of [14] which consists in minimizing the distortion on the inverse tone mapped HDR is extended in $[17,18]$ by trading this distortion against the distance between the tone mapped signal and a reference SDR version. In [18], the weighting between the MSE of the inverse tone mapped HDR signal and the SDR MSE leads to a varying quality of the SDR signal depending on the input HDR signal. In addition, most previous works (except for [18]), do no consider a 
scalable scheme in which the HDR residue is encoded when optimizing the TMO. The inverse tone mapped HDR is considered to be of sufficient quality. However, this is not the case when the base SDR layer is represented on only 8bits. Compression artifacts generated in the SDR content may be amplified depending on the slopes values of the TMO. In [18], the tone mapping curve is optimized in order to minimize a total (base plus enhancement layers) bit rate under a constraint on a distance between the uncoded tone mapped SDR video and a reference SDR version.

In this paper, we search for a new TMO that optimizes the total distortion of the HDR scalable coding scheme by taking the rate of both the base and enhancement layers into account, under a constraint on the quality of the compressed SDR signal. We first show that, given a constraint on the sum of the SDR and HDR rates, at high rates, the optimal TMO that minimizes the HDR distortion only depends on the SDR signal rate. It is then shown that this optimization problem can be decomposed into two consecutive minimization steps: (i)- a minimization of the enhancement layer variance depending on the TMO and the base layer bitrate; (ii)- and a minimization of the total HDR distortion depending on the rate repartition between the base and the enhancement layers.

The remainder of this paper is organized as follows. Section 2 presents the SDR backward compatible HDR coding model considered in this paper. The tone mapping optimization problem is formalized in Section 3 and the solution is expressed in Section 4. Section 5 presents experimental results. Finally, the conclusions are drawn in Section 6

\section{MODEL}

In this section, we model the SDR backward compatible compression of HDR content with two layers. The HDR frame consists of a sequence of luminance and chrominance values (one per pixel). Therefore, we model the HDR signal as a

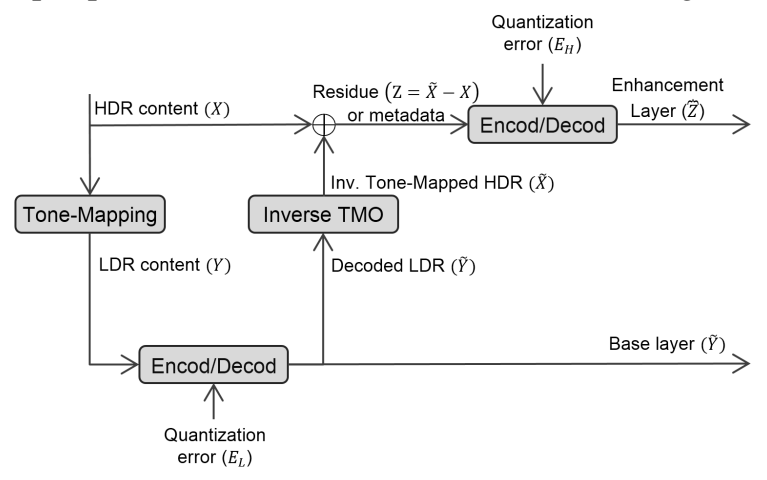

Fig. 1. Backward-compatible HDR compression scheme.

random process denoted $X$ that generates sequences of luminance (or chrominance) values. For ease of presentation, and when there is no ambiguity, we omit the pixel index and use the same notation for a random process and one random variable of the process. Then, the HDR signal is tone mapped (1) and leads to a SDR signal with smaller dynamic range (See Fig.1). The SDR signal is also modeled as a random process, denoted $Y$. The TMO, denoted $F$ is global (one HDR value $X$ leads to one SDR value $Y$ ) and is invertible such that $F^{-1}$ exists. The SDR is then compressed and this leads to a distorted SDR denoted $\tilde{Y} . E_{L}$ represents the quantization error and is defined by (2).

As for the enhancement layer, the distorted SDR signal is first inverse tone mapped to yield a distorted HDR signal $\tilde{X}$ (3), and a residue $Z$ is computed (4). Finally, this residue is compressed and the quantization error on the residue is $E_{H}$, (5). Therefore, the encoding process can be summarized as follows

$$
\begin{aligned}
Y & =F(X) \\
E_{L} & =Y-\tilde{Y} \\
\tilde{X} & =F^{-1}(\tilde{Y}) \\
Z & =X-\tilde{X} \\
E_{H} & =Z-\hat{Z}
\end{aligned}
$$

The displayed video depends on which screen is available at the receiver and on which bitstreams are received. If the only available screen is SDR, then the displayed video is $\tilde{Y}$. If the available screen is HDR, but only the SDR bitstream has been received (due to bandwidth limitation for instance), then the displayed video is $\tilde{X}$. Finally, if the viewer receives both SDR and HDR bitstreams and has an HDR display, then the displayed video is

$$
\hat{X}=\tilde{X}+\hat{Z} \text {. }
$$

\section{PROBLEM STATEMENT AND ASSUMPTIONS}

The goal is to find the TMO $F$ that minimizes the distortion of the HDR signal for a given rate budget. Indeed, the base and enhancement layers are encoded at rate $R_{S D R}$ and $R_{H D R}$ and the optimization is performed under a global rate budget $R_{T}$ i.e. under the constraint that $R_{S D R}+R_{H D R} \leq R_{T}$. The R-D function being decreasing [21, Ex10.5] with the rate $R$, the minimum distortion is obtained when the rate is maximal i.e. when the rate budget is fully utilized. Therefore, the rate inequality constraint can be turned into an equality constraint.

As in [17] and [18], this tone mapping optimization is performed while providing an SDR signal that preserves the perception and artistic intent of the HDR content. This constraint is modeled as a distortion between the compressed SDR signal $\tilde{Y}$ and an SDR obtained with another TMO $G$ that preserves the perception and artistic intent. On the whole the optimization problem can be stated as:

$$
\min _{F, R_{S D R}, R_{H D R}} D_{H D R}
$$

$$
\text { s.t. }\left\{\begin{array}{l}
D_{S D R \_R E F} \leq D_{0} \\
R_{S D R}+R_{H D R}=R_{T} \\
R_{S D R} \geq 0, R_{H D R} \geq 0
\end{array}\right.
$$

where

$$
\begin{aligned}
D_{H D R} & =\mathbb{E}\left[(X-\hat{X})^{2}\right] \\
D_{S D R \_R E F} & =\mathbb{E}\left[(G(X)-\tilde{Y})^{2}\right]
\end{aligned}
$$


The originality of the proposed work lies in the fact that (7) explicitly takes into account the compression of the enhancement layer. Indeed, this encoding is neglected in [18] since the HDR distortion is measured between the original $(X)$ and inverse tone mapped $(\tilde{X})$ HDR signal. First, this causes a mismatch since the TMO is optimized in order to have the best inverse tone mapped HDR $\tilde{X}$, which differs from the HDR signal that is available at the receiver $\hat{X}$. Second, the rate to encode the enhancement layer is not used as a parameter that can be optimized in order to perform a rate allocation between the base and enhancement layer.

We now detail the distortion terms involved in problem (7). First, the R-D performance for the compression of the SDR signal (2) and of the HDR residue (5) is well approximated by:

$$
\begin{aligned}
& \mathbb{E}\left[(Y-\tilde{Y})^{2}\right]=\alpha \sigma_{Y}^{2} 2^{-2 R_{S D R}} \\
& \mathbb{E}\left[(Z-\hat{Z})^{2}\right]=\beta \sigma_{Z}^{2} 2^{-2 R_{H D R}}
\end{aligned}
$$

where $\sigma_{Y}^{2}$ and $\sigma_{Z}^{2}$ are the variances of the SDR signal $Y$ and of the residue $Z$, and where $\alpha$ and $\beta$ are constants depending on the type of distribution and quantizer. Indeed, this model holds for many quantizers and in particular for high rate scalar quantization with variable length codes of i.i.d. but also Markovian processes [22, Chap. 5]. Therefore, we have used this model as an approximation for the compression of data with HEVC although HEVC is not strictly a scalar quantizer.

We further assume that the quantization error is independent of the compressed signal. This is a usual assumption, merely made for convenience.

Now, by construction of the two-layer compression scheme, we have that the quantization error on the residue equals the error on the HDR signal. Indeed, from (4) and (6), we have $X-\hat{X}=Z-\hat{Z}$. Therefore, from (8) and (11)

$$
D_{H D R}=\mathbb{E}\left[(Z-\hat{Z})^{2}\right]=\beta \sigma_{Z}^{2} 2^{-2 R_{H D R}}
$$

Following the first order Taylor expansion of the function $F$ as in [16], we have at high rates (i.e. when the error $E_{L}$ takes small values)

$$
Z=X-F^{-1}\left(F(X)+E_{L}\right) \approx-E_{L} F^{-1 \prime}(F(X))=\frac{-E_{L}}{F^{\prime}(X)}
$$

where' stands for the first derivative. From the independence between the residue $E_{L}$ and $X$, and assuming that $E_{L}$ has zero mean, we have

$$
\sigma_{Z}^{2}=\mathbb{E}\left[E_{L}^{2}\right] \mathbb{E}\left[F^{\prime}(X)^{-2}\right]
$$

Moreover, the encoding of $Y$ (11) yields

$$
\begin{aligned}
\mathbb{E}\left[E_{L}^{2}\right] & =\alpha \sigma_{Y}^{2} 2^{-2 R_{S D R}} \\
\text { with } \sigma_{Y}^{2} & =\mathbb{E}\left[(F(X)-\mathbb{E}[F(X)])^{2}\right]
\end{aligned}
$$

Finally,

$$
\begin{aligned}
D_{H D R}= & \alpha \beta \mathbb{E}\left[(F(X)-\mathbb{E}[F(X)])^{2}\right] \\
& * \mathbb{E}\left[F^{\prime}(X)^{-2}\right] 2^{-2\left(R_{S D R}+R_{H D R}\right)}
\end{aligned}
$$

Similarly, the distortion between the SDR signal and the reference can be derived as

$$
\begin{aligned}
D_{S D R_{-} R E F}= & \mathbb{E}\left[(G(X)-F(X))^{2}\right] \\
& +\alpha \mathbb{E}\left[(F(X)-\mathbb{E}[F(X)])^{2}\right] 2^{-2 R_{S D R}}
\end{aligned}
$$

Lemma 1. Consider a backward compatible compression scheme to encode a HDR signal into two layers (SDR and HDR), while preserving a small distortion between the SDR signal and a reference. A rate constraint is added on the sum of the rates for the SDR and HDR layers. At high rates, the optimal tone mapping $\tilde{F}$ that minimizes the HDR distortion only depends on the rate needed to encode the SDR signal.

Proof. From (15) and the sum rate equality constraint $R_{S D R}+R_{H D R}=R_{T}$, the optimization problem (7) is equivalent to first optimizing the tone mapping for each $R_{S D R}$

$$
\begin{aligned}
\tilde{F}_{R_{S D R}}= & \arg \min _{F} \mathbb{E}\left[(F(X)-\mathbb{E}[F(X)])^{2}\right] \mathbb{E}\left[F^{\prime}(X)^{-2}\right] \\
& \text { s.t. } \mathbb{E}\left[(G(X)-F(X))^{2}\right] \\
& +\alpha \mathbb{E}\left[(F(X)-\mathbb{E}[F(X)])^{2}\right] 2^{-2 R_{S D R}} \leq D_{0}
\end{aligned}
$$

and then finding the optimal rate allocation:

$$
\begin{aligned}
& \min _{R_{S D R}} \mathbb{E}\left[\left(\tilde{F}_{R_{S D R}}(X)-\mathbb{E}\left[\tilde{F}_{R_{S D R}}(X)\right]\right)^{2}\right] \mathbb{E}\left[\tilde{F}_{R_{S D R}}^{\prime}(X)^{-2}\right] \\
& \text { s.t. } 0 \leq R_{S D R} \leq R_{T}
\end{aligned}
$$

The first optimization problem (17) is equivalent to the problem solved in $[14,17,18]$,i.e. it searches to minimize the variance $\sigma_{Z}^{2}$, but with a different model.

\section{SOLUTION}

To solve the optimization problem (17-18), the tone mapping function $F(x)$ is approximated by a continuous piecewise linear function (19), as in [14, 17, 18].

$$
F(x)=\sum_{k=1}^{N}\left[\left(x-x_{k}\right) s_{k}+y_{k}\right] 1_{x \in\left[x_{k}, x_{k+1}[\right.},
$$

where $1_{x \in\left[x_{k}, x_{k+1} \text { [ }\right.}$ is the indicator function for the interval $\left[x_{k}, x_{k+1}[\right.$. To ease the optimization, we aim at reducing the number of unknowns. Therefore, the boundaries of each linear part $\left\{x_{k}\right\}_{k=1 \ldots N+1}$ of the functions are equally spaced with interval $\delta$. The values $x_{1}, x_{N+1}, \delta$ are chosen such as to span the whole range of the input HDR signal and are therefore fully determined once the number of unknowns is fixed. The minimum and maximum values of $y$ are chosen such as to span to the whole range of the SDR output. Therefore, $y_{1}=0$ and $y_{N+1}=2^{n b}$ (with $n b$ the number of SDR bits). Finally, the tone mapping (19) is

$$
\begin{aligned}
& F(x)= \\
& \sum_{k=1}^{N}\left(\left(x-x_{1}-(k-1) \delta\right) s_{k}+\delta \sum_{j=1}^{k-1} s_{j}\right) 1_{x \in\left[x_{1}+(k-1) \delta, x_{1}+k \delta[\right.}
\end{aligned}
$$

which leads to a representation where the unknowns are $\left\{s_{k}\right\}_{k=1 \ldots N}$. Using this parameterization, and under the simplification $\mathbb{E}[x] \approx \frac{x_{k+1}+x_{k}}{2}$ for each slope, the optimization problem (17-18) becomes (21). This problem can be solved numerically with the interior-point optimization method. 


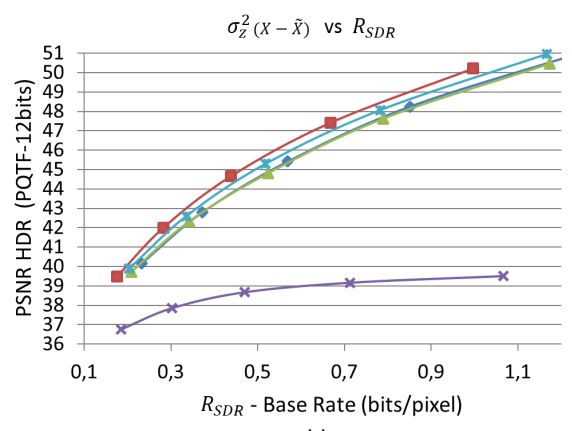

(a)

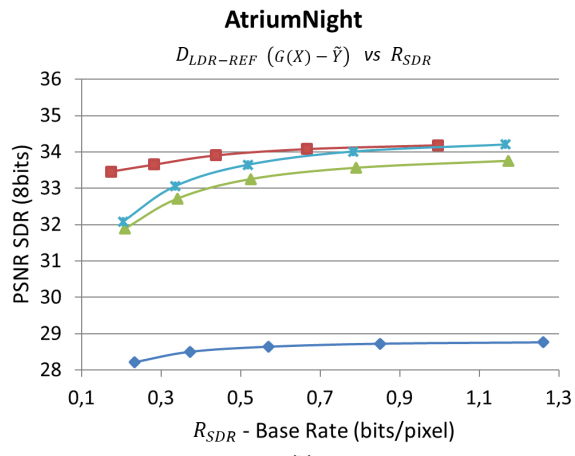

(b)

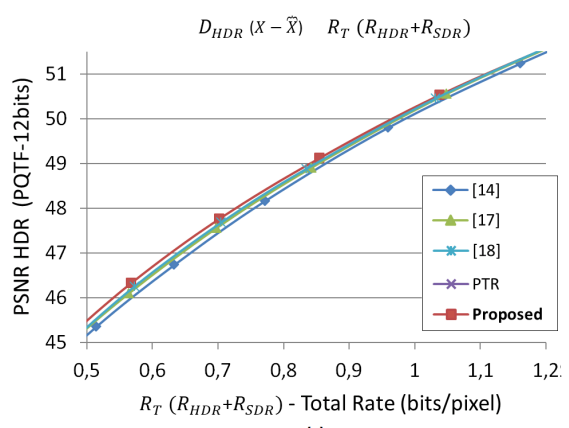

(c)

Fig. 2. R-D performance of the proposed TMO compared to other TMOs. (a): Distortion $(X-\tilde{X})$ vs rate of the base layer - (b): Distortion between SDR reference and tone-mapped images $(G(X)-\tilde{Y})$ vs rate of the base layer - (c): Distortion $(X-\hat{X})$ vs rate of both layers

$$
\begin{array}{cc}
\min _{R_{S D R}} \min _{\left\{s_{k}\right\}} & \sum_{k=1}^{N}\left(c\left(s_{k}\right)-\sum_{k=1}^{N} c\left(s_{k}\right) \cdot p_{x}(k)\right)^{2} p_{x}(k) \sum_{k=1}^{N}\left(\frac{p_{x}(k)}{s_{k}^{2}}\right) \\
\text { s.t. } \quad \alpha 2^{-2 R_{S D R}} \sum_{k=1}^{N}\left(c\left(s_{k}\right)-\sum_{k=1}^{N} c\left(s_{k}\right) p_{x}(k)\right)^{2} p_{x}(k) & \\
& +\sum_{k=1}^{N}\left(c\left(g_{k}\right)-c\left(s_{k}\right)\right)^{2} p_{x}(k) \leq D_{0} \\
& 0 \leq R_{S D R} \leq R_{T} \\
& \sum_{k=1}^{N}\left(s_{k}\right)=\frac{y_{N+1}}{\delta} \\
\text { where } & c\left(s_{k}\right)=\frac{\delta . s_{k}}{2}+\sum_{j=1}^{k-1}\left(\delta . s_{j}\right) \\
\text { and with } \quad & p_{x}(k)=P\left(x_{k} \leq x<x_{k+1}\right)
\end{array}
$$

\section{EXPERIMENTAL RESULTS}

First, the original HDR image is transformed such that the quantization becomes uniform with respect to the human eye contrast sensitivity. More precisely, the HDR signal is perceptually transformed with SMPTE-2084 [8] and uniformly quantized to 12 bits. This builds the input HDR signal $X$. For this image, the pixel distribution is computed and one TMO is computed per SDR rate value according to (21). Then, this image $X$ is tone-mapped to produce the 8 bit base layer $Y$. The SDR image is encoded with the HEVC reference software (HM 16.2) $(18 \leq Q P \leq 34)$ and inverse tone-mapped to obtain an HDR image $\tilde{X}$.

The SDR reference signal is generated using the PTR operator proposed in [9], known to give good visual quality $[10,11]$. The $D_{0}$ constraint is adjusted to $34.2 \mathrm{~dB}(\mathrm{MSE}=25)$. Several images from [23, 24], of different sizes and dynamic ranges have been tested with different constraint value $D_{0}$ Here, we show results for the "AtriumNight" image, but similar conclusions were obtained for other images [25].

Fig.2(a) plots the PSNR of the inverse tone mapped HDR image versus the SDR image bitrate, while Fig.2(b) plots the corresponding SDR PSNR with respect to the reference. The proposed TMO gives the best R-D performance and the smallest distortion with respect to the SDR reference, especially at low bitrates. Interestingly, we observe that our optimization is easier to tune in order to meet the constraint on the SDR signal. Indeed, [18] change the constrained optimization into an unconstrained optimization, where the tuning has to be done through the lagrangian multiplier. Second, [17] and [18] solve a similar optimization problem, with however a simplified model of the variance of the residue, which leads to suboptimality and worst results in Fig.2(a).

The convergence time of the proposed algorithm is about 2s (MatLab implementation) i.e. of the same order of magnitude as [18]. We use only 20 slopes, adding more slopes does not improve the R-D performances. Initializing the algorithm with the PTR or [14] speeds up the convergence.

The second minimization aims to find the best rate allocation for the base and enhancement layers. The quality of the inverse tone mapped HDR image is improved by adding the enhancement layer $Z$, that is the residue represented on 12 bits. This residue is encoded with the HM 16.2. Multiple encodings have been performed, with different combinations of base and enhancement QPs to test different rate allocations. For each value of the sum rate $\left(R_{S D R}+R_{H D R}\right)$, the pair $\left(Q P_{S D R}, Q P_{H D R}\right)$ leading to the best PSNR is retained, thus yielding the best R-D curve for each TMO (see Fig. 2(c)).

The gap between R-D curves is reduced, compared to Fig.2(a), where only the base layer is encoded. The enhancement layer helps the least efficient TMO to improve the HDR reconstruction. However, the proposed TMO still keeps the best R-D performance although it respects a stricter constraint on the SDR perceptual quality, especially at low bitrates. The gain is around $0.1 \mathrm{~dB}$, for rates in the range $(0.5,1) \mathrm{bpp}$, compared to [17] or [18].

\section{CONCLUSION}

In this paper, we proposed a TMO that maximizes the R-D performance of an HDR scalable coding scheme, while preserving a good quality for the SDR signal. We showed that the optimization problem can be split into a rate allocation and a TMO optimization problem. The proposed method leads to the best R-D performance, in comparison to state-of-the-art methods that solve approximations of the original optimization problem. 


\section{REFERENCES}

[1] F. Banterle, A. Artusi, K. Debattista, and A. Chalmers, "Advanced High Dynamic Range Imaging: Theory and Practice”, A. K. Peters, Ltd., Natick, MA, USA, 1st edition, 2011.

[2] E. Reinhard, G. Ward, S.N. Pattanaik, P.E. Debevec, and W. Heidrich, "High Dynamic Range Imaging - Acquisition, Display, and Image-Based Lighting (2. ed.)”, Academic Press, 2010.

[3] G. Ward, "Real Pixels," in Graphics Gems II, James Arvo, Ed., pp. 80-83. Academic Press, 1991.

[4] Industrial Light and Magic, "OpenEXR, HDR Image File Format," www.openexr.com/index.html.

[5] A. Motra and H. Thoma, "An Adaptive Logluv Transform for High Dynamic Range Video Compression," in Image Processing (ICIP), IEEE International Conference on, 2010.

[6] G.W. Larson, "LogLuv Encoding for Full-gamut Highdynamic Range Images," J. Graph. Tools, pp. 15-31, 1998.

[7] A. Luthra, E. Franois, and W. Husak, "Call for Evidence (CfE) for HDR and WCG Video Coding," Tech. Rep. N15083, ISO/IEC JTC1/SC29/WG11, Geneva, Switzerland, February 2015.

[8] S. Miller, M. Nezamabadi, and S. Daly, "Perceptual Signal Coding for More Efficient Usage of Bit Codes," in SMPTE Conferences. Society of Motion Picture and Television Engineers, 2012.

[9] E. Reinhard, M. Stark, P. Shirley, and J. Ferwerda, "Photographic Tone Reproduction for Digital Images," ACM Trans. Graph, 2002.

[10] P. Ledda, A. Chalmers, T. Troscianko, and H. Seetzen, "Evaluation of Tone Mapping Operators Using a High Dynamic Range Display," ACM Trans. Graph, 2005.

[11] Y. Salih, W. bt Md-Esa, A.S. Malik, and N. Saad, "Tone Mapping of HDR Images: A Review," in Intelligent and Advanced Systems (ICIAS), International Conference on, June 2012.

[12] G. Ward and M. Simmons, "JPEG-HDR: A Backwardscompatible, High Dynamic Range Extension to JPEG," in ACM Siggraph Courses, New York, USA, 2006.

[13] M. Okuda and N. Adami, “Two-layer Coding Algorithm for High Dynamic Range Images Based on Luminance Compensation," J. Vis. Comun. Image Represent., 2007.
[14] Z. Mai, H. Mansour, R. Mantiuk, P. Nasiopoulos, R. Ward, and W. Heidrich, "Optimizing a Tone Curve for Backward-Compatible High Dynamic Range Image and Video Compression," IEEE Trans. on Image Processing, June 2011.

[15] P. Lauga, G. Valenzise, G. Chierchia, and F. Dufaux, "Improved Tone Mapping Operator for HDR Coding Optimizing the Distortion/Spatial Complexity Tradeoff," in Signal Processing Conference (EUSIPCO), Proc. of the European, Lisbon, Portugal, 2014.

[16] M. Le Pendu, C. Guillemot, and D. Thoreau, "Rate distortion optimized tone curve for high dynamic range compression," in Signal Processing Conference (EUSIPCO), Proc. of the European, Lisbon, Portugal, 2014.

[17] A. Koz and F. Dufaux, "Optimized Tone Mapping with LDR Image Quality Constraint for BackwardCompatible High Dynamic Range Image and Video Coding," in Image Processing (ICIP), IEEE International Conference on, 2013.

[18] Z. Mai, H. Mansour, P. Nasiopoulos, and R.K. Ward, "Visually Favorable Tone-Mapping with High Compression Performance in Bit-Depth Scalable Video Coding," Multimedia, IEEE Trans. on, 2013.

[19] A. Cotton, T. Borer, M. Pindoria, S. Thompson, M. Naccari, S. Schwarz, and M. Mrak, "BBCs Response to CfE for HDR Video Coding (Category 3a)," Tech. Rep. M36249, ISO/IEC JTC1/SC29/WG11, Warsaw, Poland, June 2015.

[20] S. Lasserre, F. Le Lannec, E. Francois, and T. Poirier, "Technicolor's Response to CfE for HDR and WCG (category 1) - Single Layer HDR Video Coding with SDR Backward Compatibility," Tech. Rep. M36263, ISO/IEC JTC1/SC29/WG11, Warsaw, Poland, June 2015.

[21] T.M. Cover and J.A. Thomas, "Elements of Information Theory”, Wiley-Interscience, Hoboken, New Jersey, 2nd edition, 2006.

[22] T. Wiegand and H. Schwarz, "Source Coding: Part I of Fundamentals of Source and Video Coding," Foundations and Trends in Signal Processing, 2011.

[23] Max Planck Institut Informatik, "HDR Image Gallery," resources.mpi-inf.mpg.de/hdr/gallery.html.

[24] G. Ward, "High dynamic range image examples," www.anyhere.com/gward/hdrenc/pages/originals.html.

[25] "Supplementary Pictures and Results [Online]," www.irisa.fr/temics/demos/TMOoptimization. 